SVN

Stroke \& Vascular Neurology

\title{
Inflammatory biomarkers and cerebral small vessel disease: a community- based cohort study
}

Ding-Ding Zhang, ${ }^{1}$ Yuan Cao (D) ${ }^{2}$ Jing-Yu Mu, ${ }^{2}$ Yi-Ming Liu, ${ }^{3}$ Feng Gao, ${ }^{3}$ Fei Han, ${ }^{2}$ Fei-Fei Zhai, ${ }^{2}$ Li-Xin Zhou, ${ }^{2}$ Jun $\mathrm{Ni}^{2}{ }^{2}$ Ming Yao, ${ }^{2}$ Ming-Li Li, ${ }^{4}$ Zheng-Yu Jin, ${ }^{4}$ Shu-Yang Zhang, ${ }^{5}$ Li-Ying Cui, ${ }^{2}$ Yong Shen, ${ }^{3}$ Yi-Cheng Zhu (1) ${ }^{2}$

\section{ABSTRACT}

To cite: Zhang D-D, Cao Y, Mu J-Y, et al. Inflammatory biomarkers and cerebral small vessel disease: a communitybased cohort study. Stroke \& Vascular Neurology 2022;0. doi:10.1136/svn-2021-001102

- Additional supplemental material is published online only. To view, please visit the journal online (http://dx.doi.org/10. 1136/svn-2021-001102).

D-DZ and YC contributed equally.

Received 6 May 2021

Accepted 20 January 2022

A) Check for updates

(c) Author(s) (or their employer(s)) 2022. Re-use permitted under CC BY-NC. No commercial re-use. See rights and permissions. Published by BMJ.

For numbered affiliations see end of article.

Correspondence to Dr Yi-Cheng Zhu; zhuych910@163.com
Background and purpose Although inflammation has been proposed to be a candidate risk factor for cerebral small vessel disease (CSVD), previous findings remain largely inconclusive and vary according to disease status and study designs. The present study aimed to investigate possible associations between inflammatory biomarkers and MRI markers of CSVD.

Methods A group of 15 serum inflammatory biomarkers representing a variety of those putatively involved in the inflammatory cascade was grouped and assessed in a cross-sectional study involving 960 stroke-free subjects. The biomarker panel was grouped as follows: systemic inflammation (high-sensitivity C reactive protein (hsCRP), interleukin 6 and tumour necrosis factor $\alpha$ ), endothelialrelated inflammation (E-selectin, P-selectin, intercellular adhesion molecule 1, vascular cell adhesion molecule 1 (VCAM-1), CD40 ligand, lipoprotein-associated phospholipase A2, chitinase-3-like-1 protein and total homocysteine (HCY)) and media-related inflammation (matrix metalloproteinases 2, 3 and 9, and osteopontin). The association(s) between different inflammatory groups and white matter hyperintensity (WMH), lacunes, cerebral microbleeds (CMBs), enlarged perivascular space (PVS) and the number of deep medullary veins (DMVs) were investigated.

Results High levels of serum endothelial-related inflammatory biomarkers were associated with both increased WMH volume $\left(R^{2}=0.435, p=0.015\right)$ and the presence of lacunes $\left(R^{2}=0.254, p=0.027\right)$. Backward stepwise elimination of individual inflammatory biomarkers for endothelial-related biomarkers revealed that VCAM-1 was significant for WMH ( $\beta=0.063, p=0.005)$ and $\mathrm{tHCY}$ was significant for lacunes $(\beta=0.069, p<0.001)$. There was no association between any group of inflammatory biomarkers and CMBs or PVS. Systemic inflammatory biomarkers were associated with fewer DMVs $\left(R^{2}=0.032\right.$, $p=0.006$ ), and backward stepwise elimination of individual systemic-related inflammatory biomarkers revealed that $\operatorname{hsCRP}(\beta=-0.162, p=0.007)$ was significant.

Conclusion WMH and lacunes were associated with endothelial-related inflammatory biomarkers, and fewer DMVs were associated with systemic inflammation, thus suggesting different underlying inflammatory processes and mechanisms.

\section{INTRODUCTION}

Cerebral small vessel disease (CSVD) refers to a group of heterogeneous clinical

\section{Key messages}

- Although inflammation is a candidate risk factor for cerebral small vessel disease (CSVD), the findings remain largely inconclusive.

- In our study, white matter hyperintensities and lacunes were associated with endothelial-related inflammatory biomarkers, and fewer deep medullary veins were associated with systemic inflammation.

- The distinct associations indicated that underlying different inflammatory processes and mechanisms may be involved in different subtypes of CSVD.

syndromes caused by cerebral vascular anomalies, involving the small arteries, arterioles, capillaries and venules. Because cerebral small vessels cannot be directly visualised in vivo using routine MRI techniques, CSVD is commonly characterised according to underlying brain parenchymal damage and is reflected by white matter hyperintensity (WMH), lacunes, cerebral microbleeds (CMBs) and enlarged perivascular space (PVS). Deep medullary veins (DMVs), which are small parenchymal veins located in the periventricular white matter, can be visualised on susceptibility-weighted imaging (SWI). As those veins range in diameter from tens to hundreds of microns, they are also in the scope of small vessels. ${ }^{12}$

Many studies have assessed the associations between inflammation and CSVD, and have proposed that inflammation is a candidate risk factor for CSVD. ${ }^{3-5}$ However, the findings remain largely inconclusive, and vary according to disease status and study designs. ${ }^{3}$ The inflammatory response involves many pathways, and various inflammatory biomarkers are involved and interact with one another. ${ }^{6}$ Most previous studies have only investigated the associations between a single inflammatory biomarker and CSVD MRI marker. ${ }^{3}$ Given the diversity of mechanisms underlying different MRI markers of 
CSVD, it is likely that specific biomarkers are associated with different MRI markers. Nevertheless, the evidence remains limited. Therefore, we aimed to investigate possible associations between inflammatory biomarkers and CSVD based on a Chinese community cohort using a comprehensive panel of 15 inflammatory biomarkers and common CSVD MRI markers, as well as the number of DMVs.

\section{METHODS}

\section{Participants}

The present study was a cross-sectional analysis of the Shunyi study, an ongoing community-based cohort study that commenced in 2013 in China, the details of which have been published elsewhere. ${ }^{78}$ Briefly, aiming to determine risk factors for cardiovascular and age-related diseases, the Shunyi study recruited 1586 inhabitants of Shunyi (a suburban district of Beijing) $\geq 35$ years of age between June 2013 and April 2016. In addition, multidimensional information, including demographic, clinical, blood biochemical and medical imaging data, was systematically collected using standardised methods during the baseline examination. Among potential participants, 329 refused to undergo or had contradictions to MRI examination. Thus, the current study was performed based on information from 1257 participants who underwent baseline MRI. However, potentially eligible subjects without inflammatory biomarker tests $(n=257)$ and with stroke history, fever, other active inflammatory or neoplastic disease $(n=40)$ were also excluded. Thus, 960 participants were ultimately included in the final analysis. Compared with subjects not included in the current study, those included were younger, more likely to be women, more likely to be non-smokers and non-drinkers, and exhibited higher lipid levels (online supplemental table 1). All subjects read, agreed to and provided informed written consent to participate in the present study.

\section{Basic information and covariates}

A structured questionnaire was administered to collect demographic and lifestyle information. Anthropometric measurements were performed by skilled staff. Venous blood samples were drawn from subjects as per routine protocol after an overnight fast and analysed for lipid spectrum, glucose levels and other biochemical parameters. Hypertension was defined as self-reported hypertension, treatment with antihypertensive medication, systolic blood pressure $\geq 140 \mathrm{~mm} \mathrm{Hg}$ or diastolic blood pressure $\geq 90 \mathrm{~mm} \mathrm{Hg}$. Diabetes mellitus was defined as self-reported diabetes, use of oral antidiabetic drugs or insulin, or fasting serum glucose level $\geq 7.0 \mathrm{mmol} / \mathrm{L}$. Hyperlipidaemia was defined as self-reported hyperlipidaemia, treatment with antihyperlipidaemic medication, total cholesterol $>5.2 \mathrm{mmol} / \mathrm{L}$ or low-density lipoprotein $>3.36 \mathrm{mmol} / \mathrm{L}$. Current smokers were defined as those who smoked $\geq 1$ cigarette(s) per day in the past month.

\section{Measures of serum inflammatory biomarkers}

A group of 15 serum inflammatory biomarkers, representing a variety of those putatively involved in the inflammatory cascade, including those involved in systemic inflammation (high-sensitivity C reactive protein (hsCRP), interleukin 6 (IL-6) and tumour necrosis factor alpha $(\mathrm{TNF}-\alpha)$ ), endothelial-related inflammation (E-selectin, P-selectin, intercellular adhesion molecule 1 (ICAM1 ), vascular cell adhesion molecule 1 (VCAM-1), CD40 ligand $(\mathrm{CD} 40 \mathrm{~L})$, lipoprotein-associated phospholipase A2 (Lp-PLA2), chitinase-3-like-1 protein (YKL-40) and total homocysteine (tHCY) ) and media-related inflammation (matrix metalloproteinase (MMP) 2, MMP-3, MMP-9 and osteopontin (OPN)) were investigated. Overnight fasted venous blood samples were drawn and centrifuged immediately. In addition, serum was divided into 3-5 EP (Eppendorf) tubes and stored at $-80^{\circ}$. HsCRP and tHCY levels were measured immediately at the Department of Clinical Laboratory of Peking Union Medical College Hospital, in a laboratory qualified in accordance with national standards. HsCRP and tHCY levels were determined using immune turbidimetric analysis and an enzymatic cycling method, respectively, on an automated biochemistry analyzer (AU5800, Beckman Coulter, Brea, California, USA). The other 13 biomarkers were measured using the Luminex LiquiChip method (LUMINEX $100 / 200)$ and commercially available kits from R\&D Systems (catalogue number, LXSAHM-02 for MMP-2 and MMP-9, and LXSAHM-11 for the other 11 biomarkers) in 2019. The LiquiChip system integrates flow cytometry, laser technology, digital signal processing and traditional chemical technology, and has favourable features of high throughput and high flexibility. ${ }^{9}$ The inter-assay coefficients of variation for the 13 biomarkers measured using the LiquiChip system were as follows: CD40L, 3.7\%; E-selectin, 9.4\%; ICAM-1, 3.1\%; IL-6, 4\%; Lp-PLA2, 3.9\%; MMP-3, 5.6\%; OPN, 5.8\%; P-selectin, 6.3\%; TNF- $\alpha$, 10.4\%; VCAM-1, 2.2\%; YKL-40, 5.2\%; MMP-2, $5.4 \%$; and MMP-9, 3.6\%.

\section{Brain MRI markers of CSVD}

MRI of the brain was performed using a 3 Tesla scanner (Skyra, Siemens, Erlangen, Germany). Threedimensional (3D) T1-weighted images, T2-weighted images, fluid-attenuated inversion recovery and SWI were performed. Detailed MRI protocols are summarised in online supplemental table 2. All MRI markers of CSVD were defined in accordance with the standards for reporting vascular changes on neuroimaging. ${ }^{10}$ Briefly, lacunes were defined as focal lesions ranging in size from $3 \mathrm{~mm}$ to $15 \mathrm{~mm}$ with the same signal characteristics as cerebrospinal fluid (CSF) on all sequences. Dilated PVS were defined as lesions with CSF-like signals and round, ovoid or linear shapes. The severity of dilated PVS in the basal ganglia (PVS-BG) and white matter (PVS-WM) was rated using a previously established four-level severity score on 3D T1-weighted images. ${ }^{11}$ PVS-BG and PVS-WM were classified into non-severe (degree 1 and degree 2) 
and severe (degree 3 and degree 4) status due to the small number of participants. Furthermore, we defined severe PVS, where PVS-BG or PVS-WM was severe. CMBs were defined as small, round or ovoid hypointense lesions on SWI. CMB topography was divided into strictly lobar MBs (confined to the cortex and subcortical WM) or deep or infratentorial MBs (deep or infratentorial area with or without lobar MBs) location. Grey matter (GM), WM and CSF were automatically segmented on structured T1-weighted images using Statistical Parametric Mapping 12 (http://www.fil.ion.ucl.ac.uk/spm/) and CAT12 toolbox (http://www.neuro.uni-jena.de/vbm/). ${ }^{12}$ Total intracranial volume (TIV) was computed as the sum of the volumes of GM, WM and CSF. WMH volume was automatically segmented by the lesion growth algorithm as implemented in the lesion segmentation tool (LST) toolbox (http://www.statistical-modelling.de/lst.html) for Statistical Parametric Mapping 12. DMVs were visually counted in the periventricular WM of each hemisphere on reconstructed SWI. Details of the method are reported elsewhere. ${ }^{1}$ Briefly, a region of interest (ROI), $60 \times 10 \mathrm{~mm}$ in size, was delineated in the periventricular WM between the frontal and occipital horn in each cerebral hemisphere. The minimum intensity projection was derived from four consecutive slices of SWI in this region, and contoured from the ventricle floor to the superior roof of the corpus callosum, to produce a single two-dimensional image. Veins that traversed perpendicularly to the lateral ventricular and crossed the ROI were quantified by visual inspection. The number of DMVs was defined as the average count in both hemispheres.

Trained physicians who were blinded to all clinical data rated these brain MRI markers. Intra-rater agreement was assessed using a random sample of 50 individuals, with >1-month intervals between the first and second readings. The results of the intra-rater agreement analysis were as follows: the intraclass correlation coefficient was 0.68 for the number of DMVs and $\kappa$ coefficient was 0.95 for lacunes; 0.90 for CMBs; 0.71 for PVS-BG and 0.61 for PVS-WM. For automated segmentation of WMH using the LST toolbox, the Dice coefficient was 0.62 at threshold $\kappa=0.15$ when compared with manual segmentation.

\section{Statistical analyses}

Natural logarithmic (ln) transformation was performed on inflammatory biomarkers that had a skewed distribution and WMH volume, which was also skewed. Additionally, all 15 biomarkers were standardised to eliminate the influence of unique and extreme observations. Descriptive analyses were performed using the mean and SD or median (25th-75th percentile (ie, IQR)) for continuous variables, as appropriate, and frequency and percentage for categorical variables. Aiming to limit the number of comparisons and to decrease the risk for type I error, the biomarkers were divided into three groups: systemic inflammation (hsCRP, IL-6 and TNF- $\alpha$ ), endothelial-related inflammation (E-selectin, P-selectin, ICAM-1, VCAM-1, CD40L, Lp-PLA2, YKL-40 and tHCY) and media-related inflammation (extracellular matrix) (MMP-2, MMP-3, MMP-9 and OPN) based on previous studies. $^{13}$

In the primary analyses, a global omnibus test based on the regression model assessed whether at least one inflammatory biomarker in the three individual groups was significantly associated with CSVDs (WMH volume, CMBs, lacune, PVS and DMVs). General linear or logistic regression models were fitted for continuous or categorical CSVD variables, respectively. A partial $\mathrm{F}$ test was used for general linear models and a likelihood ratio test for logistic models was used to assess the significance of adding a group of inflammatory biomarkers to an existing regression model, with the $\mathrm{df}$ equal to the number of inflammatory biomarkers in the given group. ${ }^{14} 15$ To address confounding bias in an observational study, covariates were adjusted step by step based on clinical knowledge and previous studies. In model 1 , age and sex were adjusted; in model 2, age, sex, current smoking, hypertension, diabetes mellitus and hyperlipidaemia were adjusted. For WMH volume and DMVs, TIV was additionally adjusted. For the omnibus test, which was significant at the 0.05 level in fully adjusted models, regression analyses were additionally performed to force in all covariates of a given group of inflammatory biomarkers and then selecting those that could remain in the model with fully covariate-adjusted significance levels of 0.01 using the backward elimination method. In the secondary analyses, the associations between inflammatory biomarkers and CMBs and PVS according to topography (strictly lobar MBs, deep or infratentorial MBs, PVS-WM and PVS$\mathrm{BG}$ ) were tested using the same method as the primary analysis.

To account for multiple testing in the combinations of CSVDs and inflammatory biomarker group analyses, a two-tailed $p$ of $<0.01$ was defined as a statistically significant association in the primary and secondary analyses. This was a compromise to avoid type I and type II errors due to the small sample size. All statistical analyses were performed using SAS V.9.4, and conducted among complete records without missing values.

\section{RESULTS}

A total of 960 subjects was included in the analyses. The mean $( \pm \mathrm{SD})$ age of the subjects was $56.02 \pm 9.18$ years, and $35.83 \%$ (344/960) were men. Hypertension, diabetes mellitus, hyperlipidaemia and current smoking status were present in $512(53.39 \%), 157$ (16.35\%), $483(50.31 \%)$ and $210(22.41 \%)$ subjects, respectively. Detailed information regarding basic characteristics, inflammatory biomarkers and CSVD MRI markers in the study sample are summarised in table 1.

\section{Association between inflammatory biomarkers and WMH} volume

The median WMH volume is $0.9 \mathrm{~L}$ (IQR, $0.26-2.90 \mathrm{~L}$ ). The group of endothelial-related-but not systematic 
Table 1 Characteristics of the study participants

All participants

$\mathrm{n}=960$

\begin{tabular}{ll}
\hline Clinical characteristics & \\
Age, mean \pm SD, years & $56.02 \pm 9.18$ \\
Female, $\mathrm{n}(\%)$ & $616(64.17 \%)$ \\
\hline Body mass index, mean \pm SD, $\mathrm{kg} / \mathrm{m}^{2}$ & $26.55 \pm 3.80$ \\
SBP, mean \pm SD, $\mathrm{mm} \mathrm{Hg}$ & $133.63 \pm 19.27$ \\
\hline DBP, mean \pm SD, mm Hg & $78.72 \pm 10.82$ \\
\hline Current smoker, $\mathrm{n}(\%)$ & $210(22.41 \%)$ \\
\hline Hypertension, $\mathrm{n}(\%)$ & $512(53.39 \%)$ \\
\hline Diabetes mellitus, $\mathrm{n}(\%)$ & $157(16.35 \%)$ \\
\hline Hyperlipemia, $\mathrm{n}(\%)$ & $483(50.31 \%)$ \\
\hline MRI markers of CSVD assessment & \\
\hline Lacunes, $\mathrm{n}(\%)$ & $155(16.15 \%)$ \\
\hline Severe PVS, $\mathrm{n}(\%)$ & $215(22.40 \%)$ \\
\hline Severe PVS in BG, $\mathrm{n}(\%)$ & $125(13.08 \%)$ \\
\hline Severe PVS in WM, $\mathrm{n}(\%)$ & $134(14.02 \%)$ \\
\hline CMBs, $\mathrm{n}(\%)$ & $99(10.31 \%)$ \\
\hline Strictly lobar MBs, ${ }^{*} \mathrm{n}(\%)$ & $50(5.49 \%)$ \\
\hline Deep or infratentorial MBs, $\dagger \mathrm{n}(\%)$ & $49(5.38 \%)$ \\
\hline WMH volume, median $(25$ th and & $0.9(0.26,2.90)$ \\
75th percentiles), mm ${ }^{3}$ & \\
\hline Numbers of DMVs, mean $\pm S D$ & $18.86 \pm 1.70$ \\
\hline
\end{tabular}

Biomarkers of inflammation

hsCRP, median (25th and 75th

percentiles), $\mathrm{mg} / \mathrm{L}$

IL-6, median (25th and 75th

percentiles), $\mathrm{pg} / \mathrm{mL}$

TNF- $\alpha$, median $(25$ th and 75th $\quad 39.95(33.19,46.45)$

percentiles), $\mathrm{pg} / \mathrm{mL}$

E-selectin, median (25th and 75th $25.91(14.99,39.86)$

percentiles), $\mathrm{ng} / \mathrm{mL}$

P-selectin, median (25th and 75th $43.41(35.50,52.14)$

percentiles), $\mathrm{ng} / \mathrm{mL}$

ICAM-1, median (25th and 75th 294.64 (193.77,

percentiles), $\mathrm{ng} / \mathrm{mL}$

$544.98)$

VCAM-1, median (25th and 75th

percentiles), $\mathrm{ng} / \mathrm{mL}$

752.54 (585.54,

1005.20)

Lp-PLA2, median (25th and 75th

percentiles), $\mathrm{ng} / \mathrm{mL}$

$75.43(57.77,96.95)$

YKL-40, median (25th and 75th

percentiles), $\mathrm{ng} / \mathrm{mL}$

CD40L, median (25th and 75th

percentiles), $\mathrm{ng} / \mathrm{mL}$

tHCY, median (25th and 75th

percentiles), $\mu \mathrm{mol} / \mathrm{L}$

MMP-2, median (25th and 75th

percentiles), $\mathrm{ng} / \mathrm{mL}$ 189.61)

Continued
Table 1 Continued

All participants

$\mathrm{n}=960$

MMP-3, median (25th and 75th $9.79(6.20,14.94)$

percentiles), $\mathrm{ng} / \mathrm{mL}$

MMP-9, median (25th and 75th

percentiles), $\mathrm{ng} / \mathrm{mL}$

OPN, median (25th and 75th

percentiles), $\mathrm{ng} / \mathrm{mL}$

$91.12(62.34,135.87)$

$19.09(14.93,24.78)$

Missing data: body mass index, 24; SBP, 4; DBP, 4; current smoker, 23; hypertension, 1; CMBs in lobar only and any deep, 49; severe PVS in BG or WM, 4; WMH volume, 98; and DMVs, 117.

${ }^{*} \mathrm{CMBs}$ confined to the cortex and subcortical WM.

†CMBs located in deep or infratentorial areas with or without lobar MBs.

BG, basal ganglia; CD40L, CD40 ligand; CMBs, cerebral microbleeds; CSVD, cerebral small vessel disease; DBP, diastolic blood pressure; DMVs, deep medullary veins; hsCRP, highsensitivity $\mathrm{C}$ reactive protein; ICAM-1, intracellular adhesion molecule 1; IL, interleukin; Lp-PLA2, lipoprotein-associated phospholipase A2; MMP, matrix metalloproteinases; OPN, osteopontin; PVS, perivascular space; SBP, systolic blood pressure; tHCY, total homocysteine; TNF- $\alpha$, tumour necrosis factor $\alpha$; VCAM-1, vascular cell adhesion molecule 1 ; WM, white matter; $\mathrm{WMH}$, white matter hyperintensity; YKL-40, chitinase-3-like-1 protein.

biomarkers and media-related-inflammatory biomarkers were associated with WMH volume $(\mathrm{p}=0.008)$ in the model adjusted for age, sex and TIV (table 2). The findings were essentially unchanged when more confounding factors were adjusted $(p=0.015)$. However, the magnitude of variability increased was limited when the group of endothelial-related inflammatory biomarkers was added to the fully covariate-adjusted model (partial $\mathrm{R}^{2}=0.013$ (on a scale of $0-1)$ ). Backward elimination regression retained VCAM-1 $(\beta=0.063, p=0.005)$ in the fully covariateadjusted model as being associated with $\mathrm{WMH}$ volume.

\section{Association between inflammatory biomarkers and lacunes}

Lacunes occurred in $16.15 \%(n=155)$ of the sample. The group of endothelial-related inflammatory biomarkers was associated with lacunes $(p=0.005)$ in the primary model adjusted for age and sex. The findings were not substantively altered when more confounding factors were adjusted $(p=0.027)$, although the additional variability due to the group of inflammation biomarkers was only $2.8 \%$. Backward elimination regression retained $\mathrm{ln}$ tHCY (OR, 1.44, 95\% CI 1.20 to $1.74 ; \mathrm{p}<0.001$ ) in the fully covariate-adjusted model as being a risk factor for lacunes. More specifically, the increase in each SD in tHCY (ln scale) led to an increase of 0.44 -fold risk for lacunes.

\section{Association between inflammatory biomarkers and CMBs or} PVS

CMBs and severe PVS was present in 10.31\% ( $\mathrm{n}=99$ ) and $22.4 \%(n=215)$ of the sample, respectively. All of the global omnibus tests revealed that none of the groups of inflammatory biomarkers was associated with CMBs or 
Table 2 Associations between MRI markers and inflammatory biomarkers

\begin{tabular}{|c|c|c|c|c|c|c|c|c|c|c|c|c|}
\hline & \multicolumn{4}{|c|}{ Systemic biomarkers* } & \multicolumn{4}{|c|}{ Endothelial-related biomarkers $†$} & \multicolumn{4}{|c|}{ Media-related biomarkersł } \\
\hline & \multicolumn{2}{|c|}{ Model 1} & \multicolumn{2}{|c|}{ Model 2} & \multicolumn{2}{|c|}{ Model 1} & \multicolumn{2}{|c|}{ Model 2} & \multicolumn{2}{|c|}{ Model 1} & \multicolumn{2}{|c|}{ Model 2} \\
\hline & $\mathbf{R}^{2}$ & $P$ value & $\mathbf{R}^{2}$ & $P$ value & $\mathbf{R}^{2}$ & $P$ value & $\mathbf{R}^{2}$ & $P$ value & $\mathbf{R}^{2}$ & $P$ value & $\mathbf{R}^{2}$ & $P$ value \\
\hline Lacunes & 0.191 & 0.052 & 0.234 & 0.208 & 0.213 & 0.005 & 0.254 & 0.027 & 0.186 & 0.316 & 0.232 & 0.447 \\
\hline DMVs & 0.020 & 0.015 & 0.032 & 0.006 & 0.026 & 0.047 & 0.034 & 0.070 & 0.014 & 0.286 & 0.023 & 0.254 \\
\hline
\end{tabular}

The number of missing values for model 1 and model 2 is 98 and 121 for WMH volume, 0 and 23 for lacunes, 0 and 23 for CMBs, 0 and 23 for PVS and 117 and 140 for DMVs.

Model 1 is adjusted for age and sex; and model 2 is adjusted for age, sex, current smoking, hypertension, diabetes mellitus and hyperlipidaemia. For WMH volume and DMVs, the TIV is adjusted additionally.

*Systemic biomarkers include hsCRP, IL-6 and TNF- $\alpha$.

†Endothelial-related biomarkers include E-selectin, P-selectin, ICAM-1, VCAM-1, CD40L, tHCY, Lp-PLA2 and YKL-40. ¥Media-related biomarkers include MMP-2, MMP-3, MMP-9 and OPN.

CD40L, CD40 ligand; CMBs, cerebral microbleeds; DMVs, deep medullary veins; hsCRP, high-sensitivity C reactive protein; ICAM-1, intercellular adhesion molecule 1; IL-6, interleukin 6; Lp-PLA2, lipoprotein-associated phospholipase A2; MMP, matrix metalloproteinase; OPN, osteopontin; PVS, perivascular space; tHCY, total homocysteine; TIV, total intracranial volume; TNF- $\alpha$, tumor necrosis factor $\alpha$; VCAM-1, vascular cell adhesion molecule 1; WMH, white matter hyperintensity; YKL-40, chitinase-3-like-1 protein.

PVS at a significant level of 0.05 . In the secondary analyses, CMBs were categorised into strictly lobar MBs and deep or infratentorial MBs, and classified as severe PVS into only the BG and mixed WM according to topography. None of the inflammation biomarkers were associated with regional CMBs or regional severe PVS in the global omnibus tests (online supplemental table 3).

\section{Association between inflammatory biomarkers and DMVs}

The mean $( \pm \mathrm{SD})$ number of DMVs was $18.86 \pm 1.70$. Association analyses revealed that the group of systematic inflammatory biomarkers was associated with the number of DMVs ( $p=0.015$ ) when adjusted for age, sex, and TIV. The findings were not considerably altered when more confounding factors were adjusted $(\mathrm{p}=0.006)$. The additional variance was due to the group of systematic inflammation biomarkers being only $1.5 \%$. Backward elimination regression retained hsCRP $(\beta=-0.162, p=0.007)$ in the fully covariate-adjusted model as being associated with the number of DMVs. In other words, an increase in each SD of hsCRP (ln scale) led to a decrease of 0.162fold in the number of DMVs.

\section{DISCUSSION}

The present community-based cohort study investigated the associations between a broad panel of 15 inflammatory biomarkers and a spectrum of CSVD MRI markers. Our results suggested that, as a group, higher levels of endothelial-related inflammatory biomarkers were associated with increased $\mathrm{WMH}$ volume and the presence of lacunes, whereas no association between any inflammation group and CMBs and PVS was found. Furthermore, backward elimination of individual inflammatory biomarkers revealed that VCAM-1 was associated with $\mathrm{WMH}$ volume and tHCY was associated with lacunes, respectively. We also found that systematic inflammatory biomarkers, as a group, was associated with the number of DMVs. Additionally, fully adjusted models with backward elimination of inflammatory biomarkers revealed that hsCRP levels were negatively associated with the number of DMVs.

According to previous studies, the differential role of various inflammatory biomarkers involved in diverse inflammatory processes has been implicated in the pathophysiology of disease. CRP, IL- 6 and TNF- $\alpha$ have been considered to be generally sensitive-but non-specificbiomarkers of systemic inflammation, which is associated with chronic low-grade inflammation induced by arterial atherosclerosis. ${ }^{16}$ The expression of cellular adhesion molecules, such as P-selectin, E-selectin, ICAM-1 and VCAM-1, are enhanced by endothelial cells and stimulated by the binding of CD40L to CD40, ${ }^{17}$ and have exhibited a role in endothelial dysfunction as well as Lp-PLA2 and YKL-40. ${ }^{18} 19$ Additionally, HCY is another strong risk factor for endothelial cell activation, and also plays a role in the upregulation of adhesion molecules and proinflammatory cytokines. ${ }^{20}$ Both MMPs secreted by vascular smooth muscle and OPN have been observed to play multiple roles in tunica media remodelling. ${ }^{21}{ }^{22}$ Given that inflammatory biomarkers normally interact with one another in diverse inflammatory pathways rather than acting alone, multiple biomarkers should be tested simultaneously to assess the contribution of each biomarker. Among previous community-based investigations, only the Framingham Offspring Cohort study reported an association between CSVD and a panel of 15 biomarkers that were divided into three groups according to their potential function to limit the number of comparisons and the risk for type I error, and tested using ELISA kits, colorimetric activity methods or sandwich enzyme immunoassays. ${ }^{13}$ 
Herein, based on the distinctive mechanism and targets of biomarkers in vascular walls and the grouping method from the Framingham Cohort, we grouped 15 inflammatory biomarkers into 3 groups in our statistical analysis and tested using the Luminex LiquiChip system, a more sensitive testing method.

Our findings further support the proposed hypothesis regarding the basis of the pathophysiological mechanisms at the origin of WMH and lacunes, including endothelial failure. ${ }^{23}$ Both endothelial damage and atherosclerosis can be prominent features of an inflammatory process. In the current study, elevated levels of VCAM-1 and tHCY were found to be strongly correlated with WMH volume and lacunes, respectively. VCAM-1 is a cell surface sialoglycoprotein expressed by cytokine-activated endothelium on both large and small cerebral vessels, and mediates leucocyte-endothelial cell adhesion and signal transduction. ${ }^{24}{ }^{25}$ There is abundant evidence that VCAM- 1 has an important role in the development of atherosclerosis, and its role in endothelial dysfunction has also been confirmed. ${ }^{36}$ Consistent with our results, previous studies investigating VCAM-1 also reported significant associations with WMH in both stroke patients and ageing populations. ${ }^{26}$ Aside from VCAM-1, HCY was another strong risk factor for atherosclerosis through various mechanisms, such as impairment of vasodilation, promotion of low-density lipoprotein cholesterol oxidation and endothelial cell activation, upregulation of adhesion molecules and proinflammatory cytokines, and damage to the stability of vascular walls. ${ }^{20}{ }^{27}$ Higher blood levels of total HCY have been associated with a risk for atherosclerosis, cardiovascular disease and stroke. ${ }^{28}$ Similarly, both cross-sectional and longitudinal studies have found that higher levels of HCY were predictive of the prevalence and progression of lacunes. ${ }^{329}$ Our previous studies have also shown that HCY is a risk factor for lacunes using a Mendelian randomisation method. ${ }^{4}$ Overall, endothelial dysfunction and atherosclerosis caused by high levels of circulating VCAM-1 and HCY may bridge the connection between inflammation and lacunes.

Pathological mural changes in the intracerebral veins have been increasingly discussed as a part of CSVD since Moody et al described ventricular venous collagenosis. ${ }^{30}$ Owing to the difficulties in human pathological studies, the discontinuity or decreased number of DMVs visualised on SWI could be considered to be an imaging feature of venous collagenosis to assess the pathological changes in CSVD. ${ }^{2} 31$ Previous studies have suggested that a decreased number of DMVs was positively associated with age, whereas little is known about the potential mechanisms related to the cerebral small veins disease, or whether the number of DMVs is associated with inflammatory processes. ${ }^{1}$ Our results showed that high levels of systemic biomarkers, especially hsCRP, were negatively correlated with the number of DMVs, suggesting that an underlying mechanism mediated by inflammation was involved in the development of cerebral venule disease. The crucial role played by veins has already been established in inflammatory demyelinating disease and can be visualised on MRI vein imaging as the "central vein sign in WM lesion' ${ }^{32}$ According to previous studies investigating the blood-brain barrier, immune cells migrate across the vascular wall at the level of the postcapillary venules, ${ }^{33}$ which suggests that venules are involved in inflammatory processes in the central nervous system. However, previous studies investigating venules were mainly focused on inflammatory diseases of the brain, ${ }^{34} 35$ which differs from our study. According to evidence from an ageing population, inflammatory biomarkers have been linked to the pathogenesis of cerebral atherosclerosis through multiple mechanisms, especially those promoting endothelial dysfunction. ${ }^{36}$ Whether the association between hsCRP and the number of DMVs can also be explained by a similar mechanism warrants further investigation.

In contrast to our results, previous studies investigating inflammation and CMB counts and enlarged PVS have reported positive correlations in general populations, especially CMBs in deep regions. ${ }^{29} 37$ These associations may be explained by increased vascular permeability giving rise to enlarged PVS, and arteriolosclerosis is mainly involved in hypertensive vasculopathy resulting in deep CMBs, which are closely associated with elevated CRP levels. ${ }^{378}$ Our failure to confirm these associations, on the one hand, may be because our cohort included a relatively young population with a low prevalence of CMBs $(10.31 \%)$, while studies describing associations have reported an estimated prevalence of approximately $20 \%-25 \%^{29} 39$; on the other hand, the hypothetical causes of CMBs also include both cerebral amyloid angiopathy and arteriolosclerosis, whereas lacunes mainly relate to arteriolosclerosis. The contribution of acute or chronic inflammation to these two pathological processes is still to be clarified. ${ }^{40}$ This might be another potential explanation of the different associations between inflammatory factors and lacunes or CMBs.

Our study had several strengths, including a communitybased cohort sample free from vascular diseases and dementia, a broad panel of 15 inflammatory biomarkers tested as 3 groups simultaneously, a high-throughput and precise testing method using the Luminex LiquiChip system for inflammatory biomarkers, and comprehensive determination and adjustment of potential confounders. However, several caveats must be considered when interpreting our findings. First, due to the observational nature of this cross-sectional study, we only examined levels of inflammatory biomarkers at a single time without considering infection status, tumour and rheumatism, and we cannot draw definitive conclusions regarding causal relationships between inflammation and CSVD. Second, although we selected a broad spectrum of 15 circulating inflammatory biomarkers putatively involved in various phases of the inflammatory cascade, they do not adequately—nor necessarily-represent all inflammatory events. Additionally, we grouped 15 inflammatory biomarkers into 3 groups based on the distinctive 
mechanism and targets of biomarkers in vascular walls and the previous grouping method however, we could not group them by the difference of correlation power statistically. Third, since the evidence on DMVs as a marker of CSVD is controversial, the associations and mechanisms still need to be verified and clarified in further studies. Finally, although we limited our test time and set a significance level of 0.01 , we still could not mitigate the risk for type I error. Additionally, although there is no significant difference between included and excluded participants, selection bias is still inevitable. All the analyses were conducted among complete records, so the results might be biased if the missing values did not occur randomly.

In conclusion, results of our study support the hypothesis that inflammation contributes to the pathogenesis of CSVD, especially the crucial role of endothelial-related inflammatory biomarkers in $\mathrm{WMH}$ and lacunes, whereas differing inflammatory cascades may underlie diverse CSVD subtypes. The significant association between systemic inflammation and DMVs was intriguing and has improved our understanding of the underlying relationship between inflammation and cerebral venules. Further studies to validate our findings are warranted, and specific pathways should be given priority in interventional studies targeting inflammation for treatment of CSVD.

\section{Author affiliations}

${ }^{1}$ Central Research Laboratory, State Key Laboratory of Complex Severe and Rare Diseases, Peking Union Medical College Hospital, Chinese Academy of Medical Sciences and Peking Union Medical College, Beijing, China

${ }^{2}$ Department of Neurology, State Key Laboratory of Complex Severe and Rare Diseases, Peking Union Medical College Hospital, Chinese Academy of Medical Sciences and Peking Union Medical College, Beijing, China

${ }^{3}$ Division of Life Sciences and Medicine, Institute on Aging and Brain Disorders, The First Affiliated Hospital of USTC, University of Science and Technology of China, Hefei, China

${ }^{4}$ Department of Radiology, State Key Laboratory of Complex Severe and Rare Diseases, Peking Union Medical College Hospital, Chinese Academy of Medical Sciences and Peking Union Medical College, Beijing, China

${ }^{5}$ Department of Cardiology, State Key Laboratory of Complex Severe and Rare Diseases, Peking Union Medical College Hospital, Chinese Academy of Medical Sciences and Peking Union Medical College, Beijing, China

Contributors D-DZ and $Y C$ contributed equally. Guarantor for the overall content: Y-CZ. Study concept and design: Y-CZ, D-DZ and YC. Drafting of the manuscript: D-DZ and YC. Statistical analysis: D-DZ and J-YM. Study supervision and organisation of the project: Y-CZ, L-YC, YS, S-YZ, MY, JN and L-XZ. Supplying patients: Y-CZ, FH, F-FZ and J-YM. Technical consultant: M-LL, Z-YJ, Y-ML and FG.

Funding The study was funded by the National Key Research and Development Program of China (grant number: 2016YFC0901004), National Natural Science Foundation of China (grant number: 81971138), the Chinese Academy of Medical Sciences Innovation Fund for Medical Sciences (grant number: 2017-I2M-3008) and the Strategic Priority Research Program, 'Biological Basis of Aging and Therapeutic Strategies' of the Chinese Academy of Sciences (grant number: XDB39040300).

\section{Competing interests None declared.}

Patient consent for publication Not applicable.

Ethics approval This study involves human participants. The Shunyi study was approved by the ethical committee at Peking Union Medical College Hospital (Beijing, China; reference number: B-160). Participants gave informed consent to participate in the study before taking part.

Provenance and peer review Not commissioned; externally peer reviewed.
Data availability statement Data are available upon reasonable request. All data relevant to the study are included in the article or uploaded as supplementary information.

Open access This is an open access article distributed in accordance with the Creative Commons Attribution Non Commercial (CC BY-NC 4.0) license, which permits others to distribute, remix, adapt, build upon this work non-commercially, and license their derivative works on different terms, provided the original work is properly cited, appropriate credit is given, any changes made indicated, and the use is non-commercial. See: http://creativecommons.org/licenses/by-nc/4.0/.

\section{ORCID iDs}

Yuan Cao http://orcid.org/0000-0003-4257-6908

Yi-Cheng Zhu http://orcid.org/0000-0002-8966-1379

\section{REFERENCES}

1 Ao D-H, Zhang D-D, Zhai F-F, et al. Brain deep medullary veins on 3-T MRI in a population-based cohort. J Cereb Blood Flow Metab 2021;41:561-8.

2 De Guio F, Vignaud A, Ropele S, et al. Loss of venous integrity in cerebral small vessel disease: a 7-T MRI study in cerebral autosomal-dominant arteriopathy with subcortical infarcts and leukoencephalopathy (CADASIL). Stroke 2014;45:2124-6.

3 Low A, Mak E, Rowe JB, et al. Inflammation and cerebral small vessel disease: a systematic review. Ageing Res Rev 2019;53:100916.

4 Cao Y, Su N, Zhang D, et al. Correlation between total homocysteine and cerebral small vessel disease: a Mendelian randomization study. Eur J Neurol 2021;28:1931-8.

5 Altendahl M, Maillard P, Harvey D, et al. An IL-18-centered inflammatory network as a biomarker for cerebral white matter injury. PLoS One 2020;15:e0227835.

6 Libby P. Inflammation in atherosclerosis. Nature 2002;420:868-74.

7 Han F, Zhai F-F, Wang Q, et al. Prevalence and risk factors of cerebral small vessel disease in a Chinese population-based sample. J Stroke 2018;20:239-46.

8 Zhai F-F, Yan S, Li M-L, et al. Intracranial arterial dolichoectasia and stenosis: risk factors and relation to cerebral small vessel disease. Stroke 2018;49:1135-40.

9 Adamcova M, Šimko F. Multiplex biomarker approach to cardiovascular diseases. Acta Pharmacol Sin 2018;39:1068-72.

10 Wardlaw JM, Smith EE, Biessels GJ, et al. Neuroimaging standards for research into small vessel disease and its contribution to ageing and neurodegeneration. Lancet Neurol 2013;12:822-38.

11 Zhu Y-C, Tzourio C, Soumaré A, et al. Severity of dilated VirchowRobin spaces is associated with age, blood pressure, and MRI markers of small vessel disease: a population-based study. Stroke 2010;41:2483-90.

12 Schmidt P, Gaser C, Arsic M, et al. An automated tool for detection of FLAIR-hyperintense white-matter lesions in multiple sclerosis. Neuroimage 2012;59:3774-83.

13 Shoamanesh A, Preis SR, Beiser AS, et al. Inflammatory biomarkers, cerebral microbleeds, and small vessel disease: Framingham heart study. Neurology 2015;84:825-32.

14 Thakore AH, Guo C-Y, Larson MG, et al. Association of multiple inflammatory markers with carotid intimal medial thickness and stenosis (from the Framingham heart study). Am J Cardiol 2007;99:1598-602

15 Jefferson AL, Massaro JM, Wolf PA, et al. Inflammatory biomarkers are associated with total brain volume: the Framingham heart study. Neurology 2007;68:1032-8.

16 Regina C, Panatta E, Candi E, et al. Vascular ageing and endothelial cell senescence: molecular mechanisms of physiology and diseases. Mech Ageing Dev 2016;159:14-21.

17 Galkina E, Ley K. Vascular adhesion molecules in atherosclerosis. Arterioscler Thromb Vasc Biol 2007;27:2292-301.

18 Wang Y, Zhou B, Zhou P, et al. Association of lipoprotein-associated phospholipase A2 mass with asymptomatic cerebral artery stenosis. J Cell Mol Med 2018;22:2329-36.

19 Kjaergaard AD, Johansen JS, Bojesen SE, et al. Elevated plasma YKL-40, lipids and lipoproteins, and ischemic vascular disease in the general population. Stroke 2015;46:329-35.

20 Faverzani JL, Hammerschmidt TG, Sitta A, et al. Oxidative stress in homocystinuria due to cystathionine B-Synthase deficiency: findings in patients and in animal models. Cell Mol Neurobiol 2017;37:1477-85.

21 Kucukguven A, Khalil RA. Matrix metalloproteinases as potential targets in the venous dilation associated with varicose veins. Curr Drug Targets 2013;14:287-324. 
22 Fan F, Zhou Q, Xu Z, et al. Osteopontin in the pathogenesis of aortic dissection by the enhancement of MMP expressions. Int Heart $J$ 2019;60:429-35.

23 Moody DM, Brown WR, Challa VR, et al. Cerebral microvascular alterations in aging, leukoaraiosis, and Alzheimer's disease. Ann NY Acad Sci 1997;826:103-16.

24 Osborn L, Hession C, Tizard R, et al. Direct expression cloning of vascular cell adhesion molecule 1, a cytokine-induced endothelial protein that binds to lymphocytes. Cell 1989;59:1203-11.

25 Osborn L, Vassallo C, Benjamin CD. Activated endothelium binds lymphocytes through a novel binding site in the alternately spliced domain of vascular cell adhesion molecule-1. J Exp Med 1992;176:99-107.

26 Arba F, Giannini A, Piccardi B, et al. Small vessel disease and biomarkers of endothelial dysfunction after ischaemic stroke. Eur Stroke J 2019;4:119-26.

27 Böger RH, Bode-Böger SM, Sydow K, et al. Plasma concentration of asymmetric dimethylarginine, an endogenous inhibitor of nitric oxide synthase, is elevated in monkeys with hyperhomocyst(e) inemia or hypercholesterolemia. Arterioscler Thromb Vasc Biol 2000;20:1557-64.

28 Chrysant SG, Chrysant GS. The current status of homocysteine as a risk factor for cardiovascular disease: a mini review. Expert Rev Cardiovasc Ther 2018;16:559-65.

29 Miwa K, Tanaka M, Okazaki S, et al. Increased total homocysteine levels predict the risk of incident dementia independent of cerebral small-vessel diseases and vascular risk factors. J Alzheimers Dis 2016;49:503-13.

30 Moody DM, Brown WR, Challa VR, et al. Periventricular venous collagenosis: association with leukoaraiosis. Radiology 1995;194:469-76.
31 Bouvy WH, Kuijf HJ, Zwanenburg JJM, et al. Abnormalities of cerebral deep medullary veins on 7 Tesla MRI in amnestic mild cognitive impairment and early Alzheimer's disease: a pilot study. $J$ Alzheimers Dis 2017;57:705-10.

32 Sati P, Oh J, Constable RT, et al. The central vein sign and its clinical evaluation for the diagnosis of multiple sclerosis: a consensus statement from the North American imaging in multiple sclerosis cooperative. Nat Rev Neurol 2016;12:714-22.

33 Dyrna F, Hanske S, Krueger M, et al. The blood-brain barrier. J Neuroimmune Pharmacol 2013;8:763-73.

34 Sinnecker T, Bozin I, Dörr J, et al. Periventricular venous density in multiple sclerosis is inversely associated with T2 lesion count: a 7 Tesla MRI study. Mult Scler 2013;19:316-25.

35 Ge Y, Zohrabian VM, Osa E-O, et al. Diminished visibility of cerebral venous vasculature in multiple sclerosis by susceptibility-weighted imaging at 3.0 Tesla. J Magn Reson Imaging 2009;29:1190-4.

36 Schuett H, Luchtefeld M, Grothusen C, et al. How much is too much? interleukin- 6 and its signalling in atherosclerosis. Thromb Haemost 2009;102:215-22.

37 Hilal S, Ikram MA, Verbeek MM, et al. C-Reactive protein, plasma amyloid- $\beta$ levels, and their interaction with magnetic resonance imaging markers. Stroke 2018;49:2692-8.

38 Pantoni L, Garcia JH. Pathogenesis of leukoaraiosis: a review. Stroke 1997;28:652-9.

39 Lu Q-L, Li C, Song Y. Relationship of cerebral microbleeds to inflammatory marker levels. Neuroimmunol Neuroinflamm 2017;4:145-51.

40 Pétrault M, Casolla B, Ouk T, et al. Cerebral microbleeds: beyond the macroscope. Int J Stroke 2019;14:468-75. 